\title{
Phase Resonance in Centrifugal Fluid Machinery -A Comparison between Pump Mode and Turbine Mode Operations and a Discussion of Mechanisms of Flow Rate Fluctuation through a Stator-
}

\author{
Koichi Yonezawa $^{1}$, Shingo Toyahara ${ }^{1}$, Shingo Motoki ${ }^{1}$ \\ Hiroshi Tanaka ${ }^{2}$, Peter Doerfler ${ }^{3}$, and Yoshinobu Tsujimoto ${ }^{1}$ \\ ${ }^{1}$ Graduate school of Engineering Science, Osaka University \\ 1-3, Machikaneyama-cho, Toyonaka, Osaka, 560-8531, Japan, yonezawa@me.es.osaka-u.ac.jp \\ ${ }^{2}$ Toshiba Corporation (Retired) \\ ${ }^{3}$ R\&D Department, Andritz Hydro Ltd, Zurich, Switzerland
}

\begin{abstract}
Phase resonance in Francis type hydraulic turbine is studied. The phase resonance is a phenomenon that the pressure fluctuation in the penstock of hydraulic turbine installation can become very large when the pressure waves from each guide vane caused by the interaction with the runner vane reach the penstock with the same phase. Experimental and numerical studies have been carried out using a centrifugal fan. In the present study, comparisons between the pump mode and the turbine mode operations are made. The experimental and numerical results show that the rotational direction of the rotor does not affect characteristics of the pressure fluctuation but the propagation direction of the rotorstator interaction mode plays an important role. Flow rate fluctuations through the stator are examined numerically. It has been found that the blade passing flow rate fluctuation component can be evaluated by the difference of the fluctuating pressure at the inlet and the outlet of the stator. The amplitude of the blade passage component of the pressure fluctuation is greater at the stator inlet than the one at the stator outlet. The rotor-stator interaction mode component is almost identical at the inlet and the outlet of the stator. It was demonstrated that the pressure fluctuation in the volute and connecting pipe normalized by the flow rate fluctuation becomes the same for pump and turbine mode operations, and depends on the rotational direction on the interaction mode.
\end{abstract}

Keywords: Phase resonance, rotor-stator interaction, centrifugal fluid machinery, pump, turbine, flow rate fluctuation

\section{Introduction}

It was first pointed out by Den Hartog [1] that the pressure fluctuations in the penstock may become very large when the pressure waves from each blade channel between guide vanes of a Francis turbine caused by rotor-stator interaction reach the volute end in the same phase. This is called phase resonance. Chen [2] developed an equation for the criteria of the phase resonance for general combinations of guide vane and runner vane numbers and Doerfler [3] correlated with practical cases of hydro-turbines. Tanaka et al. [4] found from on-site unsteady pressure measurements in the volute that the phase resonance occurs when the rotational velocity of the interaction mode pattern due to rotor-stator interaction agrees with the velocity of sound. Following this finding, the authors of the present study confirmed this by experiments [5] using a small fan and developed a one-dimensional theory for the evaluation of the pressure fluctuation in the penstock. The theory was extended to include the effects of acoustic resonance and the effects were confirmed by experiments [6]. Large amplitude pressure fluctuations which might be caused by the phase resonance have reported also for pumps [7][8]. The purpose of the present study is to clarify the difference and similarity of the phase resonance in pumping and turbine operations.

\section{Experimental Method}

Air is used as the working fluid. As shown in Fig.1, the volute was connected to a silencer through a long duct of length $2120 \mathrm{~mm}=23.5 D_{2}$ with the cross section of $17.5 \mathrm{~mm} \times 40 \mathrm{~mm}$, to avoid the acoustic resonance. The other side of the silencer is connected to a settling tank with the diameter $145 \mathrm{~mm}$ and the length $750 \mathrm{~mm}$. The tank is connected to a ring blower through a flow meter and a flow control valve. Figure 2 shows the location of flush-mounted pressure transducers. Simultaneous measurements were made at least at two locations to clarify the phase relationships. 
Table 1 shows the dimensions of the rotor and stators. In order to study the effects of rotor-stator interaction, stators with the blade number $S=16$ and 20 are used combined with a rotor with the number of blades $R=6$. When the relation

$$
m=n R+k S
$$

is satisfied with arbitrary integer numbers $m, n$ and $k$, an interaction mode with

$$
\exp \left[j m\left(\frac{n R}{m}\right) t\right]
$$

appears where $\Omega$ is the angular velocity of the rotor and $\theta$ is the circumferential angle in the absolute frame. This shows that the interaction pattern rotates much faster than the rotor when $n R / m$ is large and the rotational speed of the interaction mode can become supersonic even at subsonic rotor speed.

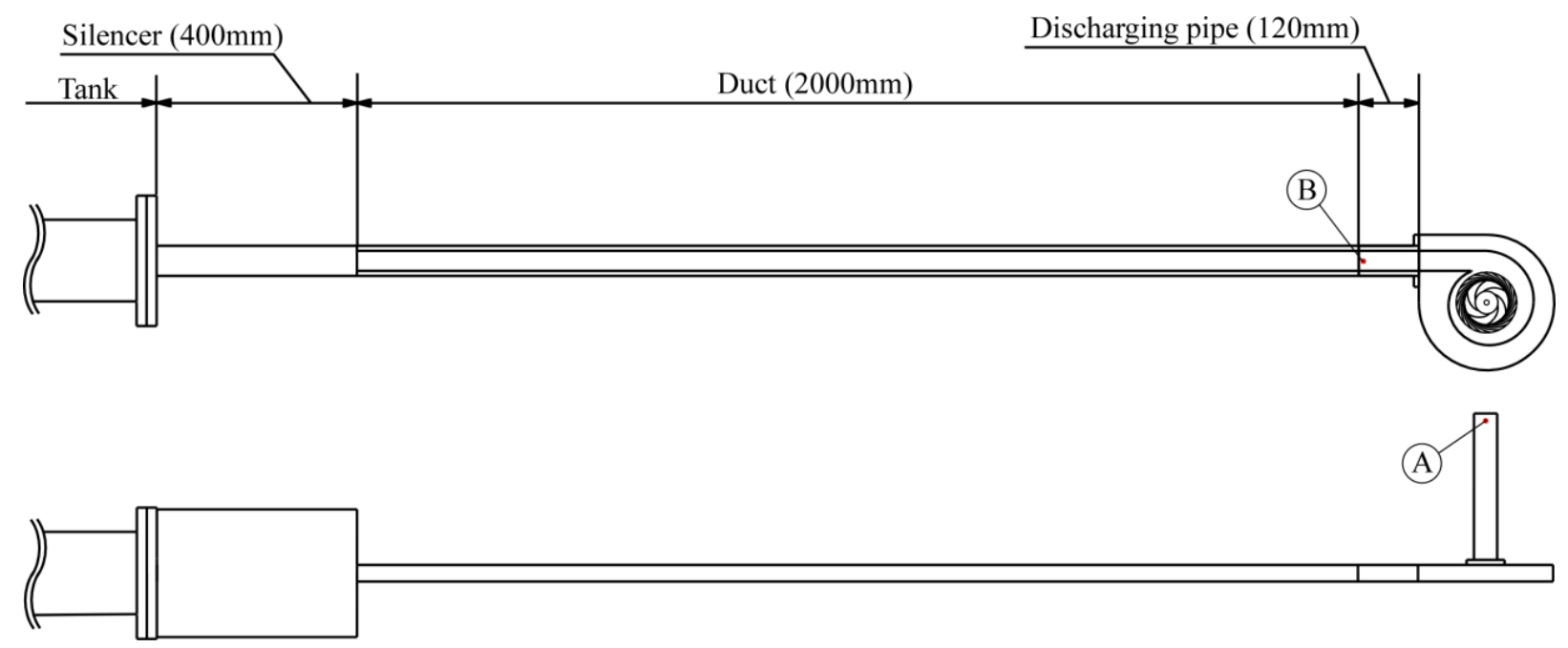

Fig. 1 Experimental facility

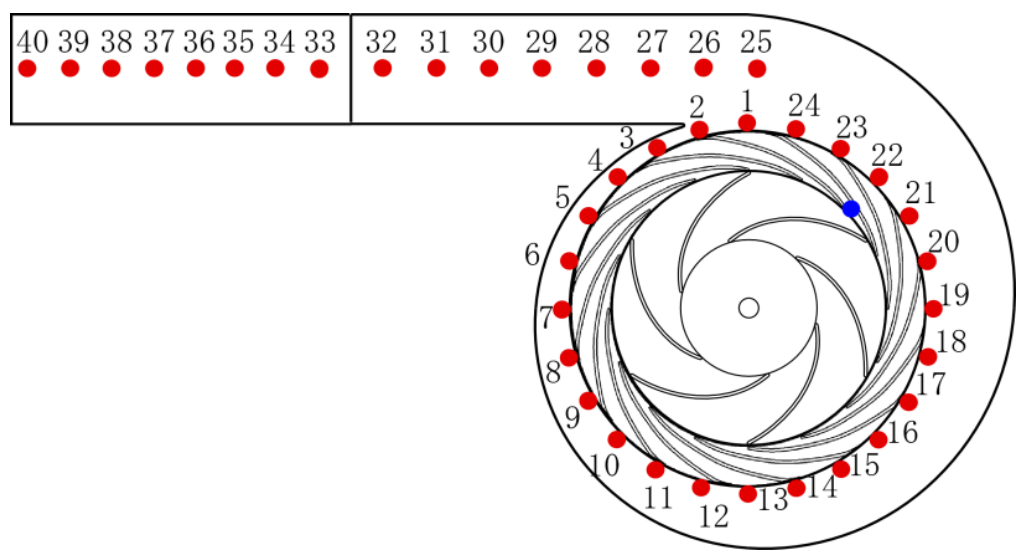

Fig. 2 Pressure sensor position 
Table 1 Dimensions of rotor and stator

\begin{tabular}{|c|c||c|c|}
\hline \multicolumn{2}{|c||}{ Rotor } & \multicolumn{2}{c|}{ Stator } \\
\hline \hline Number of vanes & 6 & Number of vanes & 16,20 \\
\hline Inlet vane angle [deg] & 22 & Inlet vane angle [deg] & 3 \\
\hline Outlet vane angle [deg] & 27.6 & Outlet vane angle [deg] & 25 \\
\hline Inner diameter [mm] & 45 & Inner diameter [mm] & 91 \\
\hline Outer diameter [mm] & 90 & Outer diameter [mm] & 121 \\
\hline Inner width [mm] & 10 & Inner width [mm] & 7.2 \\
\hline Outer width [mm] & 7.5 & Outer width [mm] & 12.5 \\
\hline Blade thickness [mm] & 1 & Blade thickness [mm] & 0.5 \\
\hline
\end{tabular}

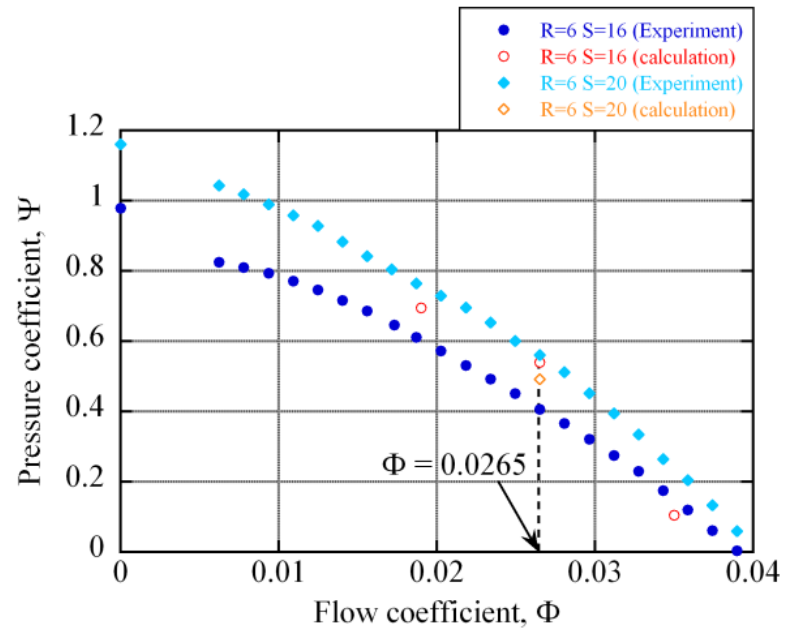

(a) Pump mode

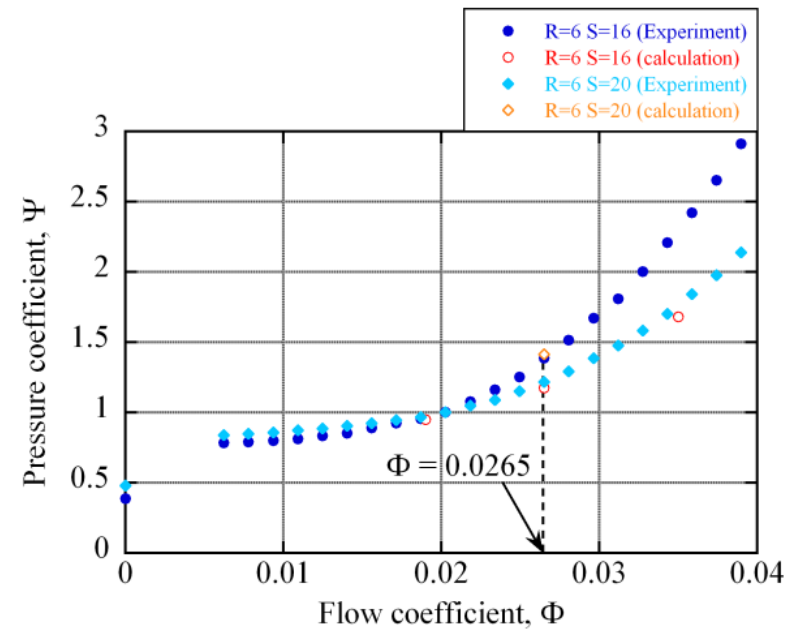

(b) Turbine mode

Fig. 3 Performance curve

\section{Numerical Analysis}

A commercial software ANSYS CFX-14.0 has been used for the analysis of unsteady compressible three-dimensional flow with SST turbulence model. About 3,400,000 cells were used for the case of $S=16$ and 4,500,000 cells for $S=20$. As the boundary conditions for the pump mode operation, the mass flow and the total temperature were specified at the inlet boundary and the static pressure condition and the non-reflecting boundary condition of Poinsot and Lele [9] were applied at the outlet. For the turbine mode operation, we failed to apply the non-reflective boundary condition at the inlet. So, we simply specified the total pressure and the total temperature at the inlet and the mass flow at the exit. The time step corresponding to $1 / 300$ of the rotational period of the rotor was used. Sub-iteration in each time step was made until the residual becomes less than $10^{-4}$ or larger than 10 iterations.

\section{Steady Performance}

Figure 3 shows the performance curves obtained at 5,600 rpm for the case with $S=16$ and at 5,300 rpm for the case with $S=20$. The flow coefficient and the pressure coefficient are defined as $\Phi=Q /\left(U_{T} D_{T}^{2}\right)$ and $\Psi=\Delta p /\left(\rho U_{T}^{2} / 2\right)$ using the outer diameter $D_{T}$ and the speed $U_{T}$ of the rotor. The difference of the pressure $\Delta p$ at $\mathrm{A}$ and $\mathrm{B}$ in Fig. 1 has been used. The disagreement between experiment and CFD might be caused by the inaccuracy of the geometrical data of the stator. The results at the flow coefficient $\Phi=0.0265$ will be discussed in the present study.

\section{Spectrum of Pressure Fluctuation in the Duct}

Figure 4 shows the spectrum of the pressure fluctuation measured at No.40 in Fig. 2, at $\Phi=0.0265$ and various rotor speed $N$. The amplitude is normalized as $\psi_{e}=\Delta p /\left(\rho U_{T}^{2} / 2\right)$. The component with the frequency $3 R N$ can be observed for all cases and we focus on this component. This occurs since the interaction mode with $2=3 \times 6-1 \times 16$ exists for $m=n R+k S$ with $R=6$ and $S=16$ and $-2=3 \times 6-1 \times 20$ with $R=6$ and $S=20$. Equation (2) shows that the interaction mode rotates in the same direction of the rotor for the case with a positive value of $\mathrm{m}$ and in the opposite direction with a negative value of $m$. Hence, the interaction mode rotates in the direction from the tongue towards the connecting pipe for the pump mode operation with $S=16$ and the turbine mode operation with $S=20$, and from the connecting pipe towards the tongue for the turbine mode operation with $S=16$ and pump mode operation with $S=20$. The results shown in Fig. 4 show that the pressure amplitude of the $3 R N$ component becomes the maximum near $N=93.3 \mathrm{~Hz}=5,600 \mathrm{rpm}$ for the case with $\mathrm{S}=16$ and near $N=88.3 \mathrm{~Hz}=5,300 \mathrm{rpm}$ for the case with $S=20$. The rotational velocity $\pi D_{4}(\mathrm{nR} / \mathrm{m}) \mathrm{N}$ of the interaction mode at the outer diameter $D_{4}$ of the guide vane becomes $319 \mathrm{~m} / \mathrm{s}$ and $302 \mathrm{~m} / \mathrm{s}$ for the cases with $S=16$ and $S=20$ respectively. The rotational speed agrees with the velocity of sound $340 \mathrm{~m} / \mathrm{s}$ at $1.06-1.13 D_{4}$ within the volute. 
This shows that the peak is caused by phase resonance. The results obtained at these speeds and at $\Phi=0.0265$ will be shown hereafter.

\section{Pressure Fluctuation in the Volute and Connecting Pipe}

Figures 5-8 shows the amplitude and the phase of $3 R N$ component in the volute and the connecting pipe. "Tongue" corresponds to No. 1 in Fig. 2 and " $L=405 \mathrm{~mm}$ " corresponds to No. 25 . With the pump mode operation with $S=16$ and turbine mode operation with $S=20$, where the interaction mode rotates in the direction from the tongue to the connecting pipe, the amplitude is nearly constant and the phase changes continuously. This is because only a mode propagating in the direction of mode rotation exists. On the other hand, with the turbine mode operation with $S=16$ and pump mode operation with $S=20$, where the interaction mode rotates in the direction from the pipe to the tongue, four loops appears and the phase changes discontinuously with a step of $180^{\circ}$. One-dimensional model [5][6] shows that this is caused by the interaction of a mode propagating towards the tongue while increasing the amplitude caused by the interaction and a propagating wave reflected from the tongue towards the pipe. The results in Figs. 6 and 7 suggest that the tongue should be treated as an open end in the present case of wide tongue clearance rather than a closed end as assumed in the theoretical model for tighter tongue clearance.

The above observations show that the direction of the interaction mode rotation is more important than the rotational direction of the rotor. Comparisons between experiment and CFD show that the pressure amplitude in the volute can be estimated by CFD but the accuracy of that in the pipe is not so good.

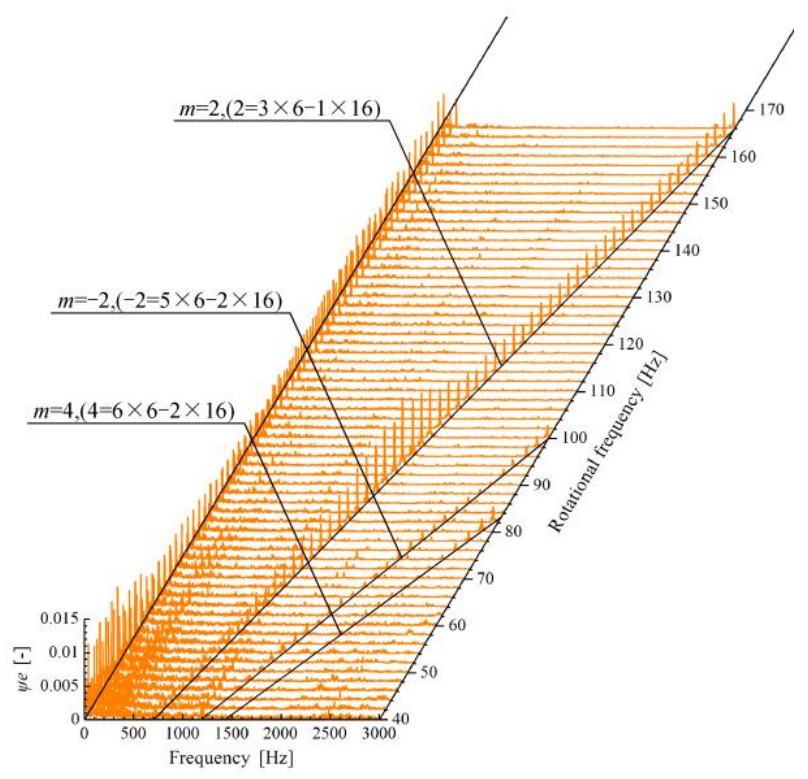

(a) Pump mode, $S=16$

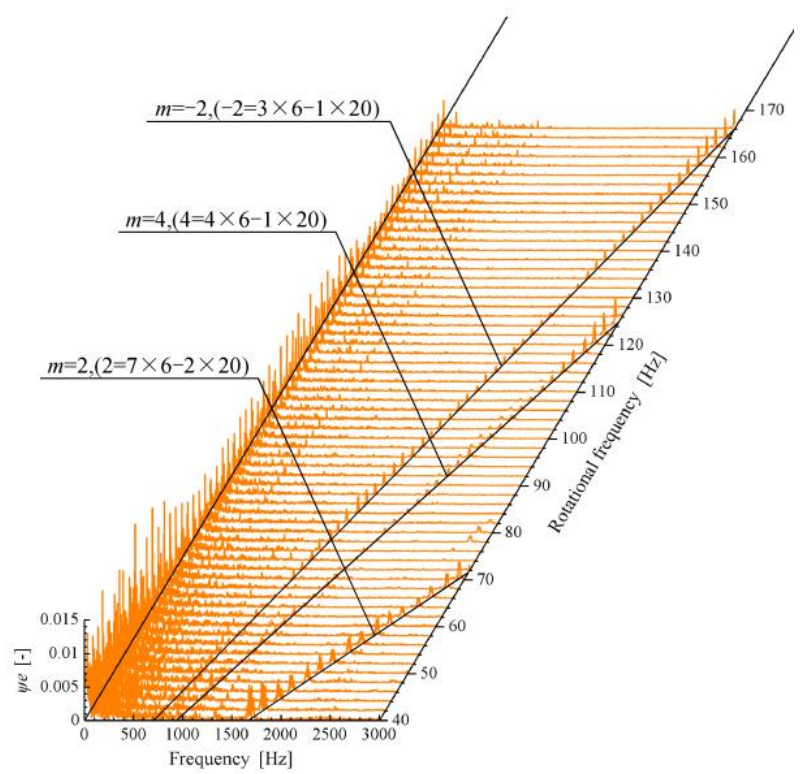

(c) Pump mode, $S=20$

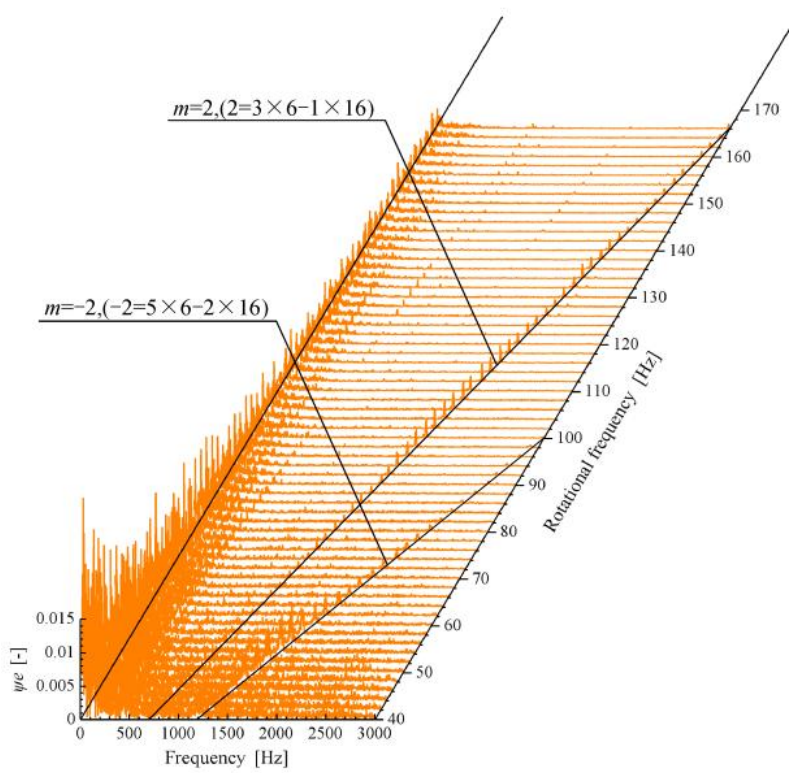

(b) Turbine mode, $S=16$

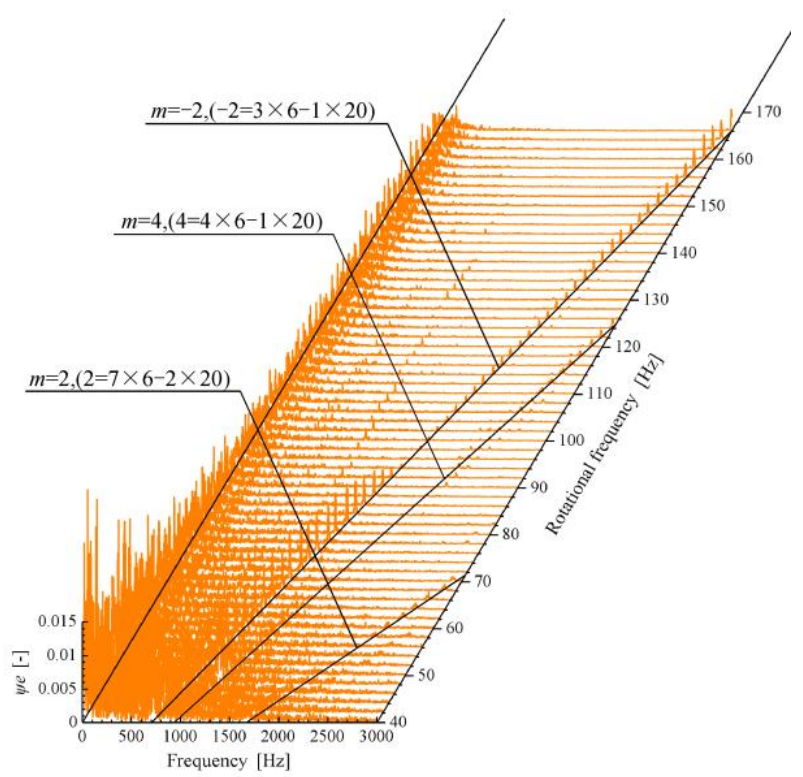

(d) Turbine mode, $S=20$

Fig. 4 Spectra of the pressure fluctuation at No. 40 pressure measurement location shown in Fig. 2, $\Phi=0.0265$ 


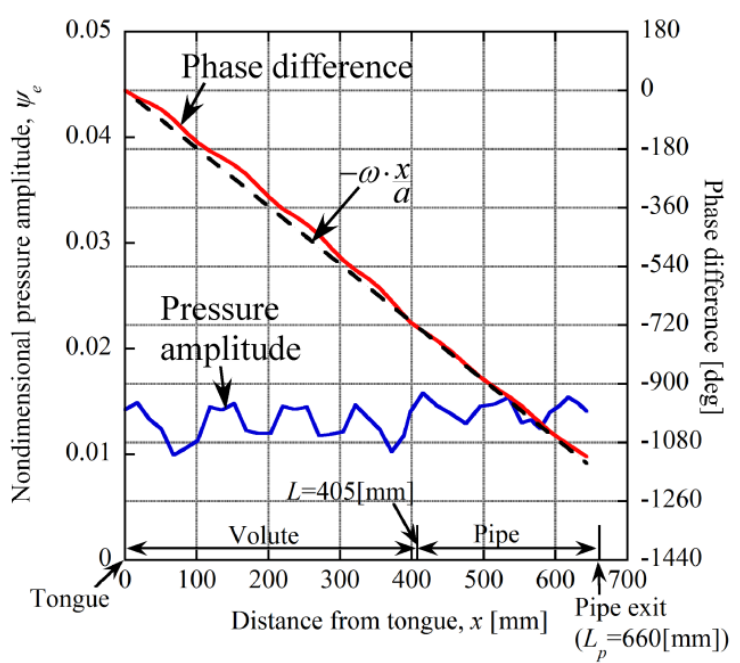

(a) Experimental result

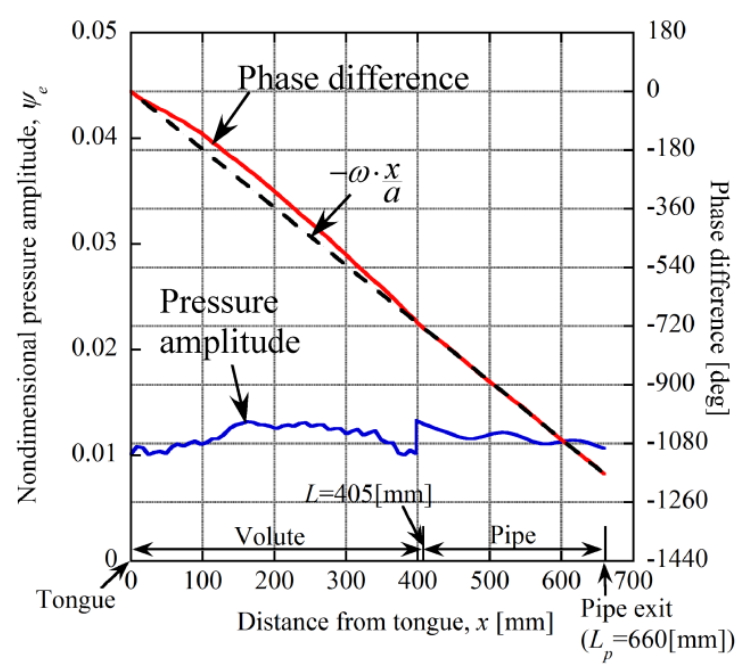

(b) Calculation result

Fig. 5 Amplitude and phase of pressure fluctuation ( $S=16$, Pump mode, 5600rpm)

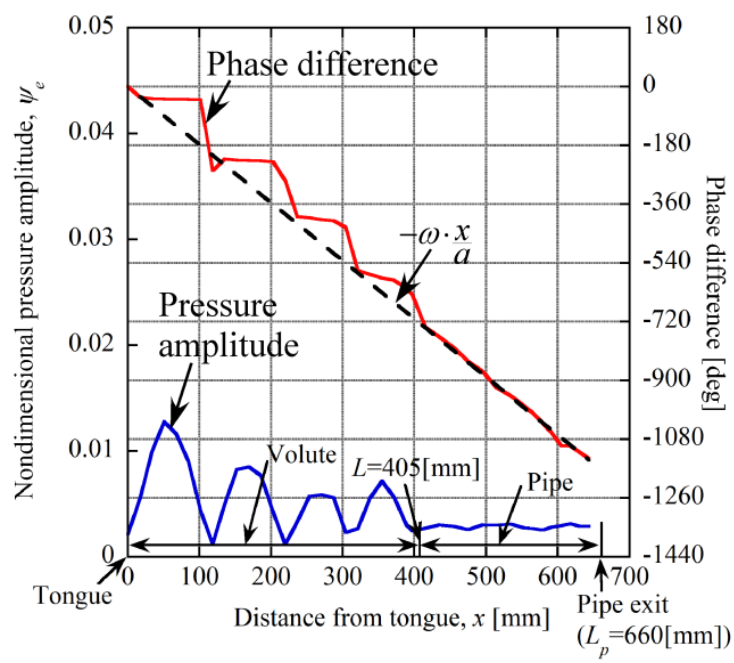

(a) Experimental result

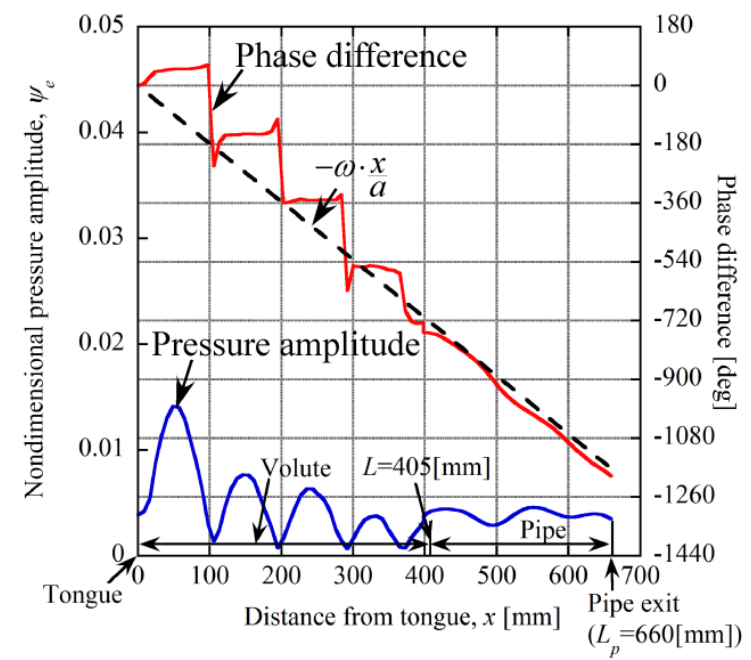

(b) Calculation result

Fig. 6 Amplitude and phase of pressure fluctuation ( $S=16$, Turbine mode, 5600rpm)

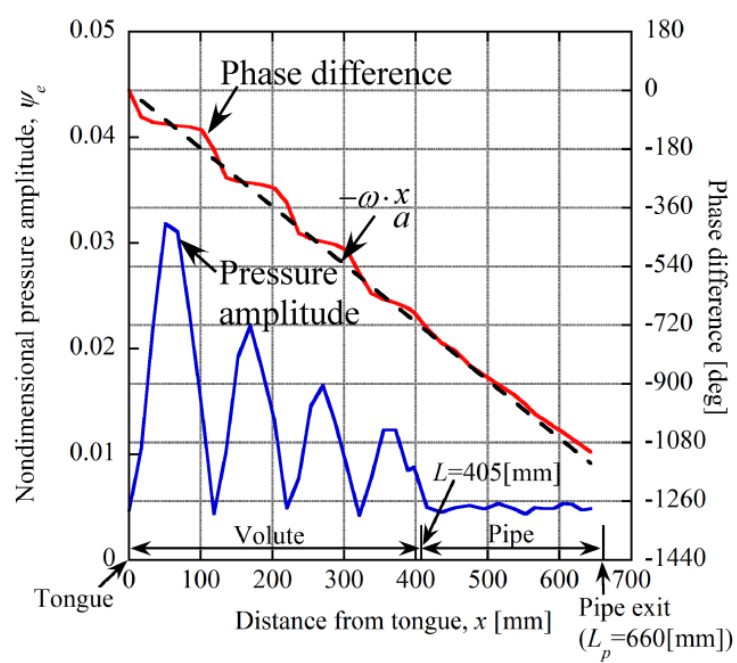

(a) Experimental result

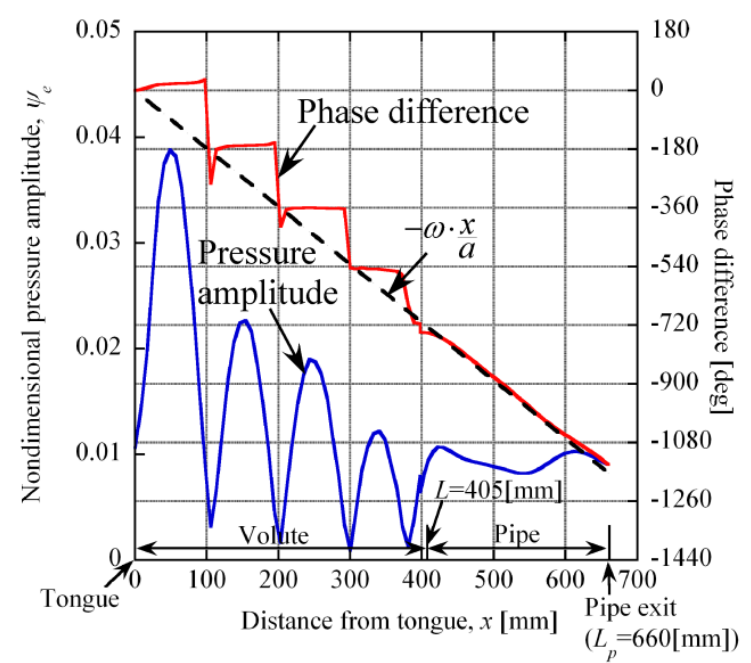

(b) Calculation result

Fig. 7 Amplitude and phase of pressure fluctuation ( $S=20$, Pump mode, 5300rpm) 


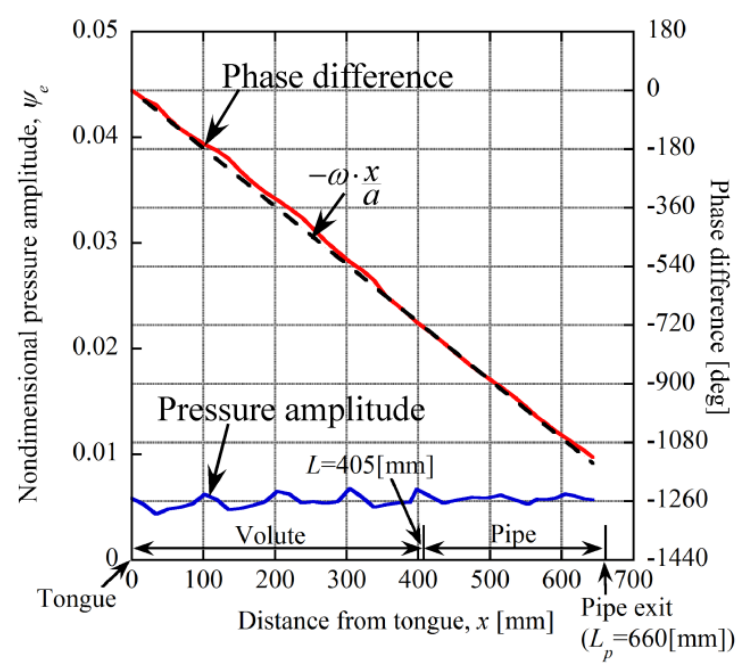

(a) Experimental result

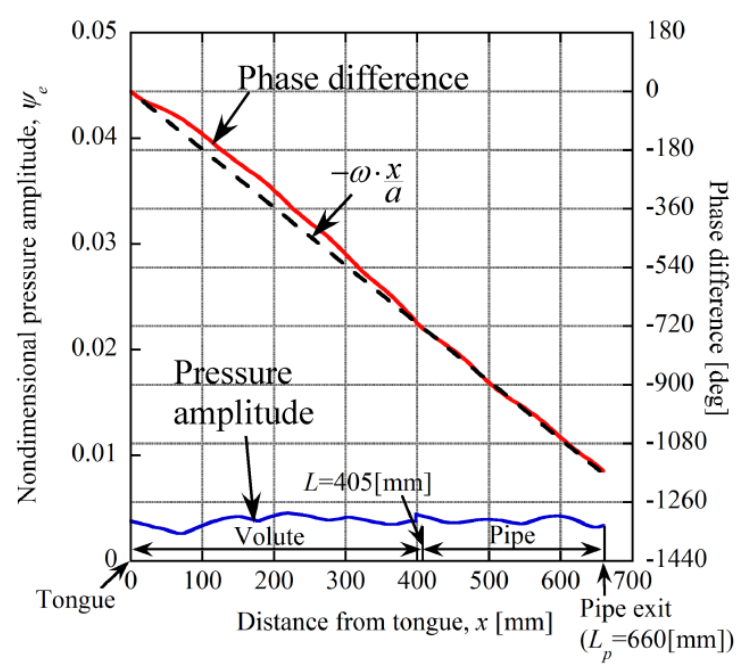

(b) Calculation result

Fig. 8 Amplitude and phase of pressure fluctuation ( $S=20$, Turbine mode, 5300rpm)

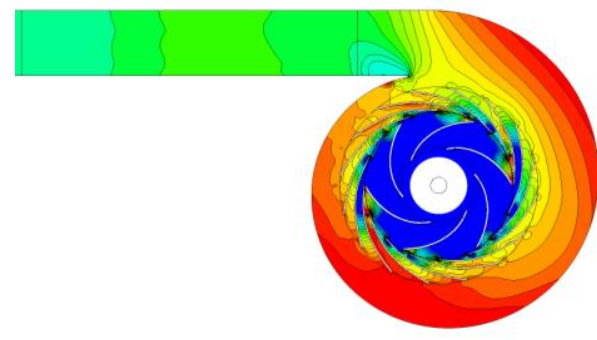

(1)

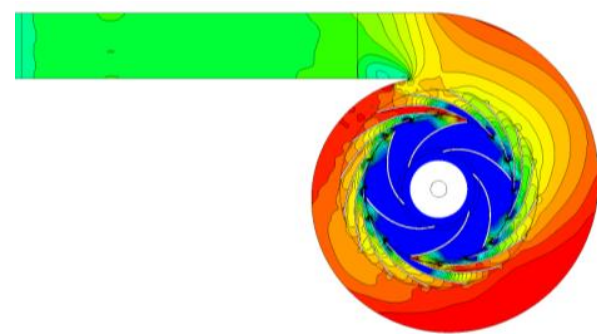

(2)

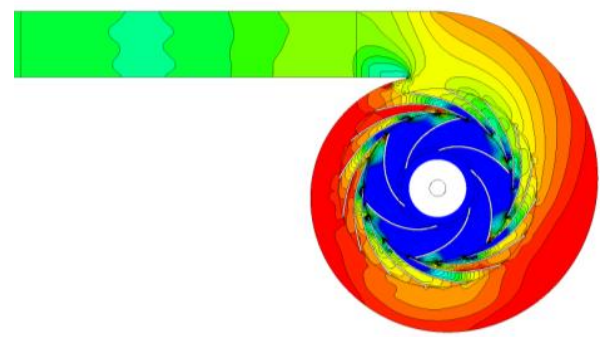

(3)(Condition(i))

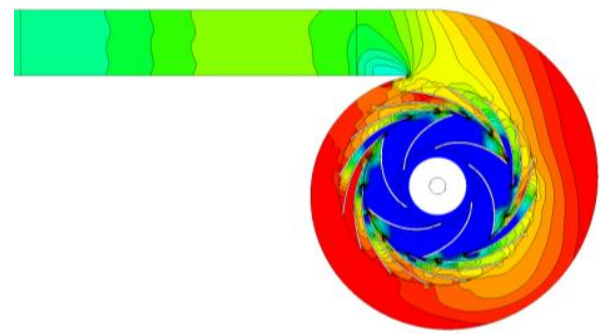

(4)

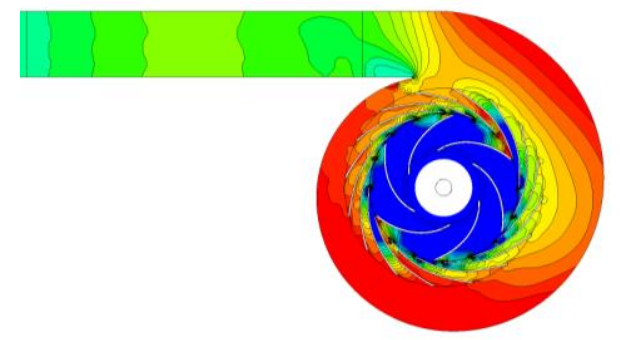

(5)(Condition(ii))

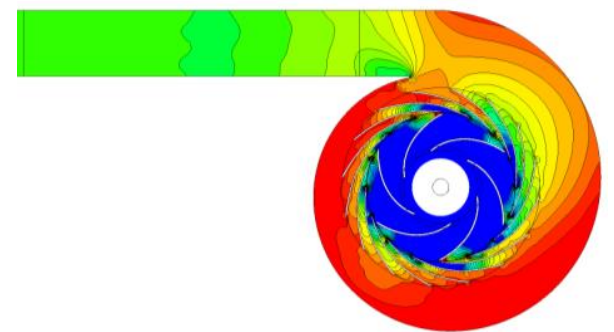

(6)

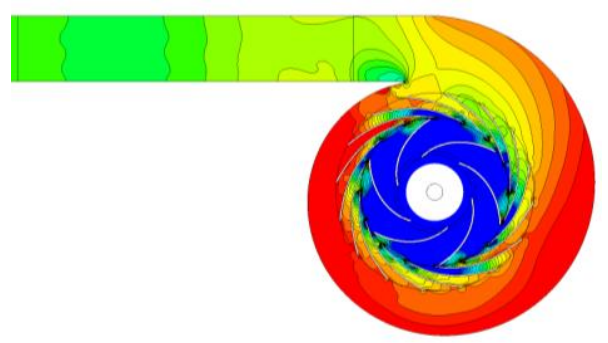

(7)(Condition(iii))

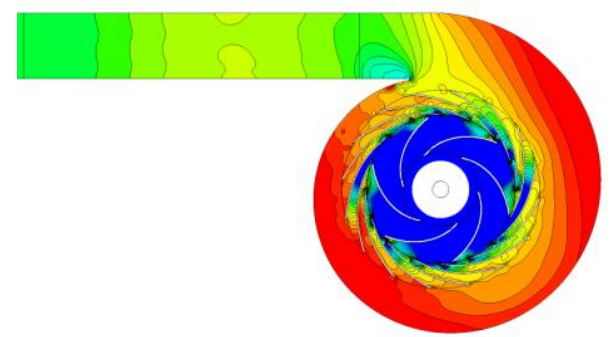

(8)

Fig. 9 Pressure distribution ( $\mathrm{R}=6, \mathrm{~S}=16$,Pump mode) 


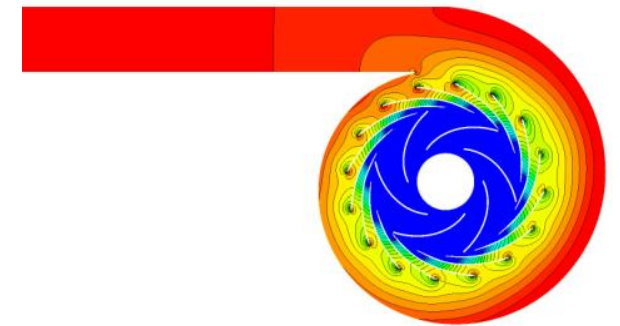

(1)

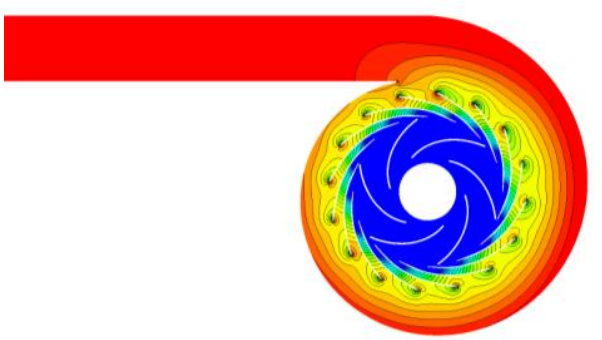

(2)

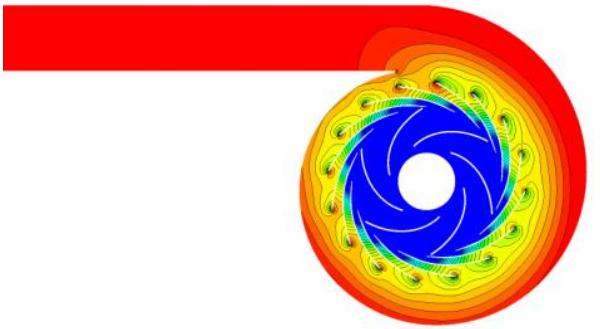

(3)(Condition(i))

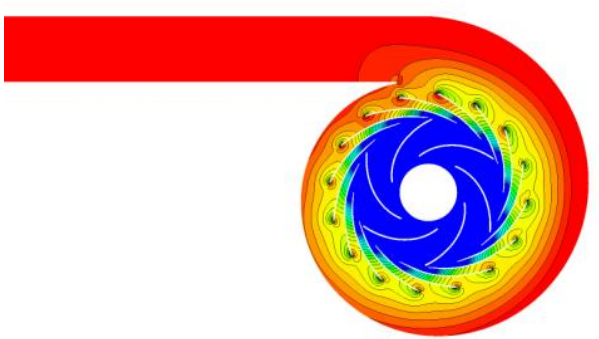

(4)

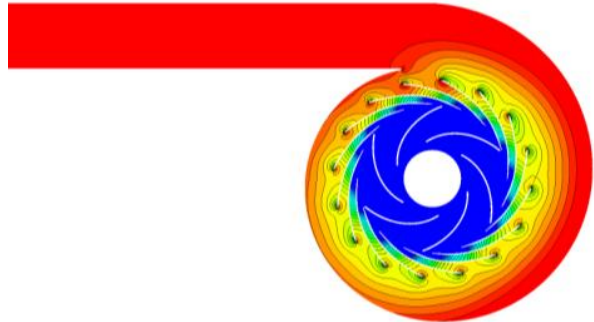

(5)(Condition(ii))

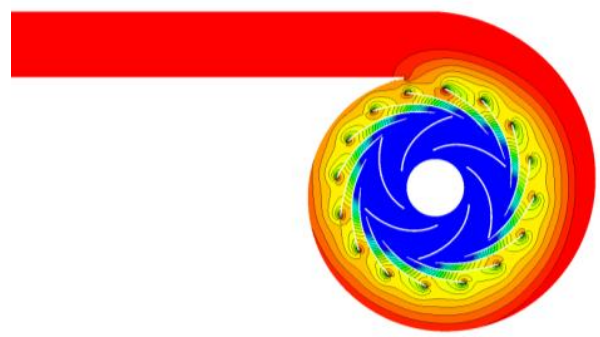

(6)

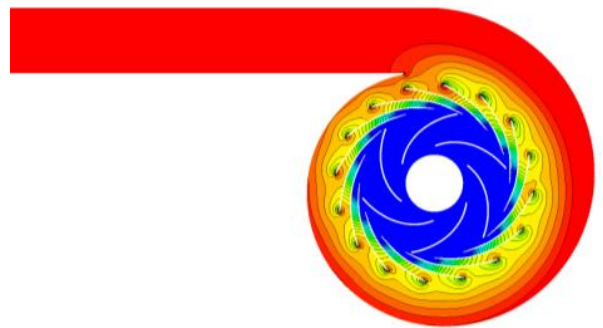

(7)(Condition(iii))

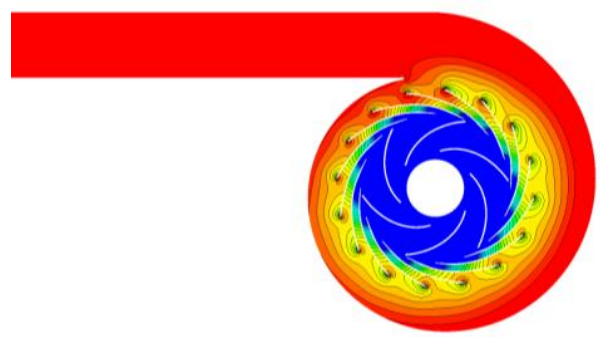

(8)

Fig. 10 Pressure distribution $(\mathrm{R}=6, \mathrm{~S}=16$, Turbine mode)

\section{Unsteady pressure field}

Figures 9 and 10 show the unsteady pressure distributions for the pump and turbine mode operations with $S=16$. With the pump mode operation, we can observe the propagation of $m=2$ mode from the tongue to the pipe. Although not very clear, we can observe a standing wave pattern with the maximums of pressure fluctuations at about $\pm 45^{\circ}$ from horizontal direction.

Details of pressure distribution around the rotor and stator blades are shown in Figs. 11 and 12. For the pump mode operation, a large pressure fluctuation occurs at the leading edge of the guide vane at the instant (ii) when the rotor blade passes by the leading edge of the guide vane. However, for the turbine mode, the pressure on the pressure surface of the rotor blade near the leading edge is slightly decreased at the instant of (ii). Although not clear from the figures, the plots of pressure distribution on the blades show, under turbine mode operation, that the pressure difference of the SS1/SS16 blade is decreased at the instant of (i)/(iii), respectively.

The gradient of circumferential pressure distribution at the outer radius of the rotor is larger for the pump mode operation than turbine mode operation leading to larger pressure fluctuation with the pump mode operation. Although not shown, plots of velocity vector shows that the velocity distribution near the inner radius of the stator is pretty flat for the turbine mode operation. However, the flow velocity near the pressure side and outer radius of the rotor is significantly decreased for the pump mode operation. This may be the cause of larger amplitude pressure fluctuation of the pump mode operation as shown in Figs. 5 and 6. 


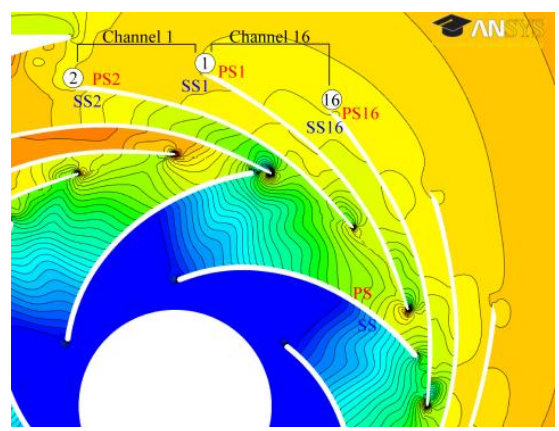

(i)

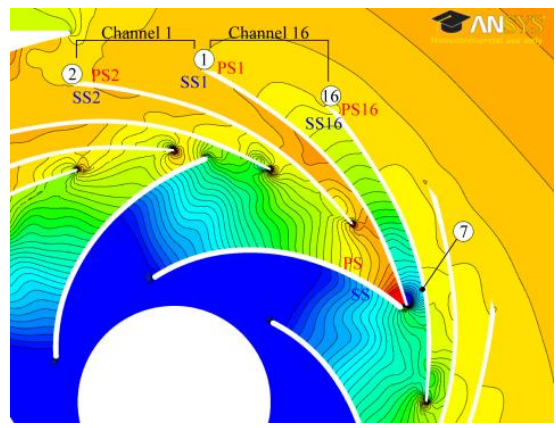

(ii)

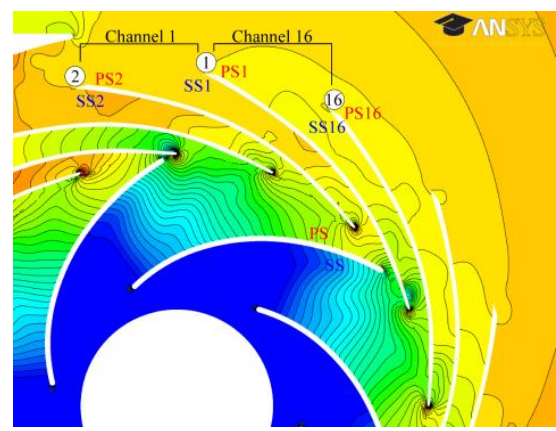

(iii)

Fig. 11 Pressure distribution, pump mode, $S=16$

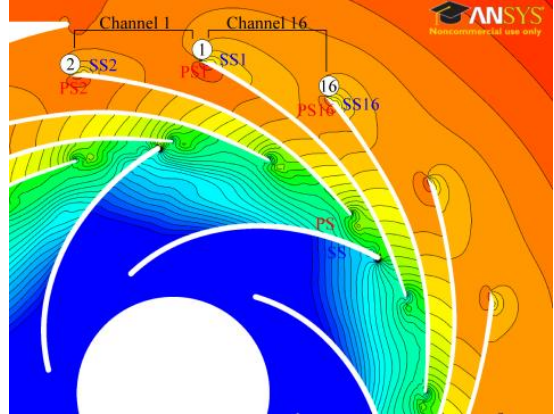

(i)

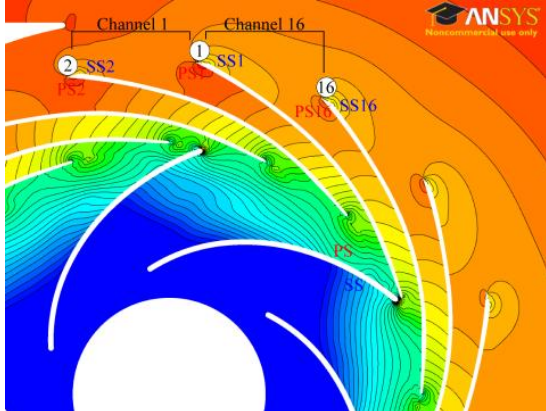

(ii)

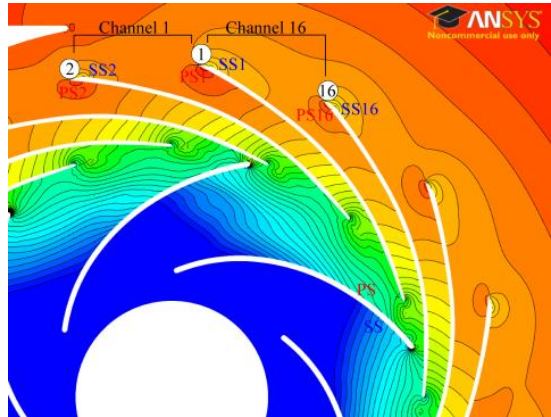

(iii)

Fig. 12 Pressure distribution, pump mode, $S=20$

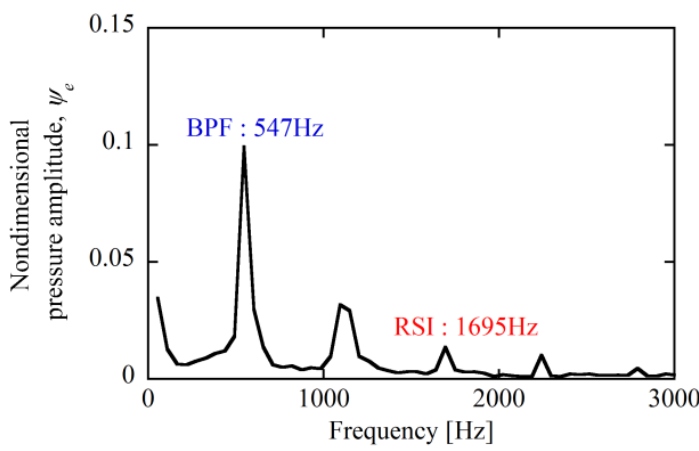

(a) Inside of stator channel

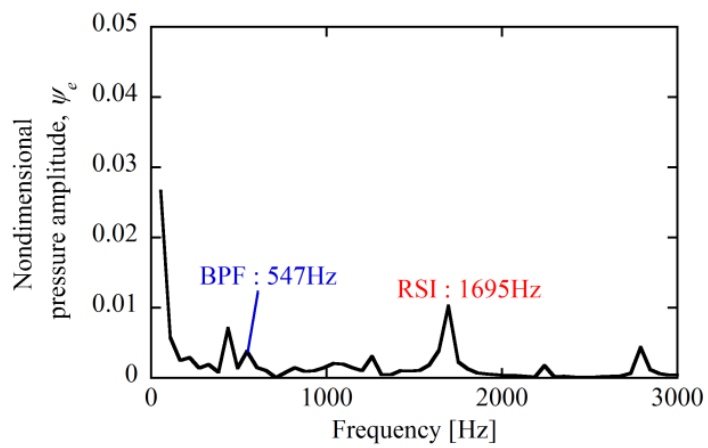

(b) Outside of stator channel

Fig. 13 Amplitude of pressure fluctuation at inside and outside of guide vanes (CFD, S=16, Pump mode)

\section{Pressure Fluctuations at the Inner and Outer Radius of Stator and Flow Rate Fluctuation in Stator Blade Channel}

In the one dimensional model [5][6], it is assumed that the flow rate fluctuation through the stator blade channel occurs with a mode of Eq. (2) due to rotor-stator interaction and causes pressure fluctuation in the volute. Here, we examine the relation between the pressure fluctuation and the flow rate fluctuation based on CFD results.

Figure 13 shows the spectra of pressure fluctuation at the inner and outer radius of the stator for the pump mode operation with $S=16$. At the inner radius, the blade passing frequency component, BPF, is dominant. However, the rotor-stator interaction component RSI with $2=3 \times 6-1 \times 16$ is dominant at the outer radius. Since the relation $6=1 \times 6-0 \times 16$ exists for the blade passing component $\mathrm{BPF}$, it can exist at the inner radius of the stator without being cancelled. At the outer radius, BPF with $m=6$ decreases significantly as compared with RSI with $m=2$. This is similar to the potential disturbance of $1 / z^{m}$ where $z$ is a complex coordinate. Similar results were obtained for other cases of pump/turbine modes and $S=16,20$.

Tables 2-5 show the amplitude of pressure fluctuations at the inner and outer radius of the stator and the flow rate fluctuation. The pressure fluctuation of the BPF component becomes significantly smaller at the outer radius. However, the magnitude of RS I component is of similar order at the inner and outer radius, except for the case shown in Table 5. As a result, RSI component is larger than the BPF component at the outer radius. The flow rate fluctuation should be the same at the inner and outer radius but some numerical error is found. The RSI component of the flow rate fluctuation is about $1 / 10$ of BPF component. In order to explain why larger RSI pressure fluctuation occurs with smaller flow rate fluctuation, Fig.14 shows the result of 1-D model: 
Table 2 Amplitude of BPF and RSI component ( $S=16$, Pump mode)

\begin{tabular}{|c||c|c|}
\hline & Inside of guide vanes & Outside of guide vanes \\
\hline \hline \multirow{4}{*}{ Pressure } & $\tilde{\psi}_{I n, B P F}=0.0992$ & $\tilde{\psi}_{\text {Out }, B P F}=0.00381$ \\
& $\tilde{\psi}_{I n, R S I}=0.0136$ & $\tilde{\psi}_{\text {Out }, R S I}=0.0103$ \\
& $\tilde{\psi}_{I n, R S I} / \tilde{\psi}_{I n, B P F}=0.137$ & $\tilde{\psi}_{\text {Out RSI }} / \tilde{\psi}_{\text {Out }, B P F}=2.70$ \\
\hline \multirow{4}{*}{ Flow rate } & $\tilde{\phi}_{I n, B P F}=5.22 \times 10^{-5}$ & $\tilde{\phi}_{\text {Out }, B P F}=5.22 \times 10^{-5}$ \\
& $\tilde{\phi}_{I n, R S I}=4.68 \times 10^{-6}$ & $\tilde{\phi}_{\text {Out } R S I}=5.96 \times 10^{-6}$ \\
& $\tilde{\phi}_{I n, R S I} / \tilde{\phi}_{I n, B P F}=0.0897$ & $\tilde{\phi}_{\text {Out }, R S I} / \tilde{\phi}_{\text {Out }, B P F}=0.114$ \\
\hline
\end{tabular}

Table 3 Amplitude of BPF and RSI component ( $S=16$, Turbine mode)

\begin{tabular}{|c||c|c|}
\hline & Inside of guide vanes & Outside of guide vanes \\
\hline \hline \multirow{3}{*}{ Pressure } & $\tilde{\psi}_{I n, B P F}=0.0236$ & $\tilde{\psi}_{\text {Out }, B P F}=0.00208$ \\
& $\tilde{\psi}_{I n, R S I}=0.00513$ & $\tilde{\psi}_{\text {Out }, R S I}=0.00406$ \\
& $\tilde{\psi}_{I n, R S I} / \tilde{\psi}_{I n, B P F}=0.217$ & $\tilde{\psi}_{\text {Out RSI }} / \tilde{\psi}_{\text {Out }, B P F}=1.95$ \\
\hline \multirow{3}{*}{ Flow rate } & $\tilde{\phi}_{I n, B P F}=8.85 \times 10^{-6}$ & $\tilde{\phi}_{\text {Out }, B P F}=8.98 \times 10^{-6}$ \\
& $\tilde{\phi}_{I n, R S I}=1.87 \times 10^{-6}$ & $\tilde{\phi}_{\text {Out }, R S I}=2.35 \times 10^{-6}$ \\
& $\tilde{\phi}_{I n, R S I} / \tilde{\phi}_{I n, B P F}=0.211$ & $\tilde{\phi}_{\text {Out }, R S I} / \tilde{\phi}_{\text {Out }, B P F}=0.262$ \\
\hline
\end{tabular}

Table 4 Amplitude of BPF and RSI component ( $S=20$, Pump mode)

\begin{tabular}{|c|c|c|}
\hline & Inside of guide vanes & Outside of guide vanes \\
\hline \multirow{3}{*}{ Pressure } & $\tilde{\psi}_{I n, B P F}=0.0316$ & $\tilde{\psi}_{\text {Out }, B P F}=0.00536$ \\
\hline & $\tilde{\psi}_{I n, R S I}=0.0103$ & $\tilde{\psi}_{\text {Out }, R S I}=0.00992$ \\
\hline & $\tilde{\psi}_{I n, R S I} / \tilde{\psi}_{I n, B P F}=0.307$ & $\tilde{\psi}_{\text {Out }, R S I} / \tilde{\psi}_{\text {Out }, B P F}=1.85$ \\
\hline \multirow{3}{*}{ Flow rate } & $\tilde{\phi}_{I n, B P F}=3.68 \times 10^{-5}$ & $\tilde{\phi}_{\text {Out }, B P F}=4.12 \times 10^{-5}$ \\
\hline & $\tilde{\phi}_{I n, R S I}=2.98 \times 10^{-6}$ & $\tilde{\phi}_{\text {Out }, R S I}=5.68 \times 10^{-6}$ \\
\hline & $\tilde{\phi}_{I n, R S I} / \tilde{\phi}_{I n, B P F}=0.0810$ & $\tilde{\phi}_{\text {Out }, R S I} / \tilde{\phi}_{\text {Out }, B P F}=0.142$ \\
\hline
\end{tabular}

Table 5 Amplitude of BPF and RSI component ( $S=20$, Turbine mode)

\begin{tabular}{|c||c|c|}
\hline & Inside of guide vanes & Outside of guide vanes \\
\hline \hline \multirow{4}{*}{ Pressure } & $\tilde{\psi}_{I n, B P F}=0.0414$ & $\tilde{\psi}_{\text {out }, B P F}=7.51 \times 10^{-4}$ \\
& $\tilde{\psi}_{I n, R S I}=0.0231$ & $\tilde{\psi}_{\text {Out }, R S I}=0.00213$ \\
& $\tilde{\psi}_{I n, R S I} / \tilde{\psi}_{I n, B P F}=0.558$ & $\tilde{\psi}_{\text {Out }, R S I} / \tilde{\psi}_{\text {Out }, B P F}=2.84$ \\
\hline \multirow{4}{*}{ Flow rate } & $\tilde{\phi}_{I n, B P F}=1.05 \times 10^{-5}$ & $\tilde{\phi}_{\text {Out }, B P F}=1.13 \times 10^{-5}$ \\
& $\tilde{\phi}_{I n, R S I}=9.29 \times 10^{-7}$ & $\tilde{\phi}_{\text {Out }, R S I}=1.11 \times 10^{-6}$ \\
& $\tilde{\phi}_{I n, R S I} / \tilde{\phi}_{I n, B P F}=0.0885$ & $\tilde{\phi}_{\text {Out }, R S I} / \tilde{\phi}_{\text {Out }, B P F}=0.0982$ \\
\hline
\end{tabular}




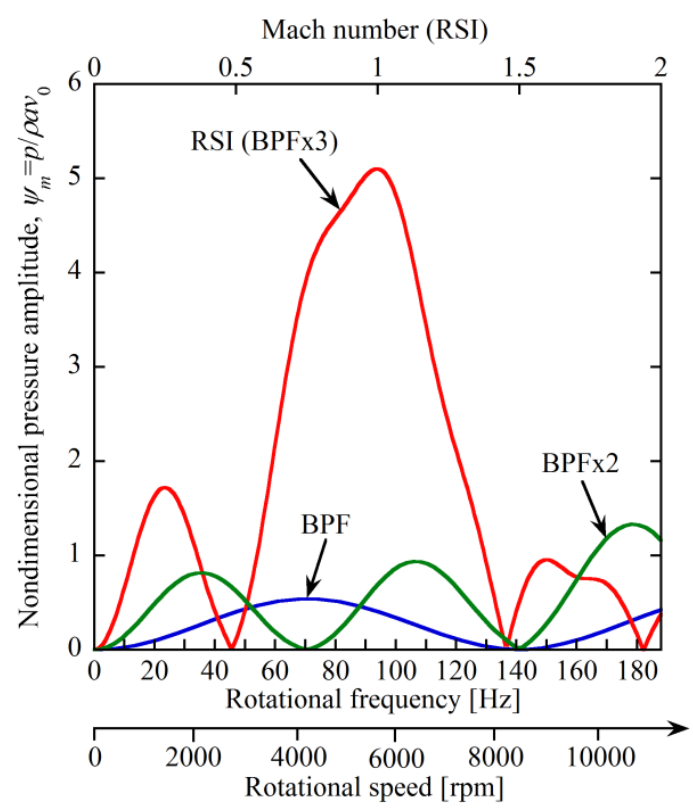

Fig. 14 Pressure fluctuation by 1-D model

Table 6 Amplitude of flow rate fluctuation evaluated from the pressure difference

\begin{tabular}{|cc|c|c|c|c|c|c|c|c|}
\hline \multirow{2}{*}{} & \multicolumn{4}{|c|}{} & \multicolumn{3}{|c|}{$S=16$} & \multicolumn{3}{c|}{$S=20$} \\
\cline { 2 - 11 } & \multicolumn{2}{|c|}{ Pump } & \multicolumn{2}{c|}{ Turbine } & \multicolumn{2}{c|}{ Pump } & \multicolumn{2}{c|}{ Turbine } \\
\cline { 2 - 11 } & BPF & RSI & BPF & RSI & BPF & RSI & BPF & RSI \\
\hline$\tilde{p}_{3}[\mathrm{~Pa}]$ & 40.90 & 5.607 & 9.730 & 0.8575 & 38.04 & 1.979 & 15.29 & 8.530 \\
\hline$\tilde{p}_{4}$ & {$[\mathrm{~Pa}]$} & 1.571 & 4.246 & 2.115 & 1.674 & 11.67 & 3.663 & 0.2773 & 0.7866 \\
\hline$\tilde{p}=\left|p_{3}-p_{4}\right|[\mathrm{Pa}]$ & 39.88 & 8.547 & 8.879 & 2.493 & 35.90 & 13.61 & 15.09 & 9.293 \\
\hline$\tilde{Q}$ & {$\left[\mathrm{~m}^{3} / \mathrm{s}\right]$} & $1.02 \times 10^{-5}$ & $7.04 \times 10^{-7}$ & $2.27 \times 10^{-6}$ & $2.05 \times 10^{-7}$ & $6.62 \times 10^{-6}$ & $8.11 \times 10^{-7}$ & $2.78 \times 10^{-6}$ & $5.53 \times 10^{-7}$ \\
\hline$\tilde{\phi}$ & {$[-]$} & $4.76 \times 10^{-5}$ & $3.29 \times 10^{-6}$ & $1.06 \times 10^{-5}$ & $9.60 \times 10^{-7}$ & $3.27 \times 10^{-5}$ & $4.01 \times 10^{-6}$ & $1.38 \times 10^{-5}$ & $2.74 \times 10^{-6}$ \\
\hline \hline$\tilde{\phi}$ (CFD) $[-]$ & $5.22 \times 10^{-5}$ & $4.68 \times 10^{-6}$ & $8.85 \times 10^{-6}$ & $1.87 \times 10^{-6}$ & $3.68 \times 10^{-5}$ & $2.98 \times 10^{-6}$ & $1.05 \times 10^{-5}$ & $9.29 \times 10^{-7}$ \\
\hline
\end{tabular}

$$
\begin{aligned}
& \frac{p}{a v_{0}}=\frac{L / b}{2 m} \frac{1}{1 M^{2}} C e^{i \frac{2 m}{L} U\left(t \frac{x}{a}\right) \div}=\frac{L / b}{2 m} \frac{1}{1 M^{2}} C e^{i \gg m M \frac{x}{L}} \times e^{i t} \\
& C=1 \quad e^{i 2 M m}+\frac{1 M}{2}\left(e^{i 2 M m} e^{i 2 M m}\right)
\end{aligned}
$$

where $v_{0}$ is the radial velocity fluctuation caused by rotor-stator interaction, $U$ and $M=U / a$ are the phase velocity and the Mach number of the interaction mode, $L$ is the length of the volute, $b$ is the width of the volute. The rotational speed of the BPF component agrees with the rotor speed $\Omega$ but the RSI component rotates with the speed of $n R \Omega / m=9 \Omega$ which results in $M=1$ around 5,600rpm where the non-dimensional amplitude $p / \rho a v_{0}$ becomes the maximum. This is phase resonance. On the other hand, rotational Mach number of the BPF component becomes one at the rotational speed of 5,600 $\times 9=50,400 \mathrm{rpm}$ and the nondimensional amplitude is very small at the speed discussed here. This explains the larger pressure amplitude of the RSI component. However, CFD at non-phase resonance condition and incompressible CFD resulted in the RSI component at the outer radius about half of those shown in Tables 2-5. This shows that the rotor-stator interaction itself can cause the RSI component at the outer radius of the stator.

\section{Evaluation of the flow rate fluctuation through the stator passage}

We can evaluate the flow rate fluctuation by assuming that the fluid in the passage between stator blade is accelerated by the pressure fluctuation at the inner and outer radius of the stator. The results are shown in Table 6. is the result thus obtained and (CFD) is the result of CFD. Reasonable agreement is obtained for the BPF component but the agreement is poor for the RSI component. We should note that the amplitude of the pressure fluctuation $\tilde{p}_{4}$ at the outer radius is non-negligible for the RSI component. 


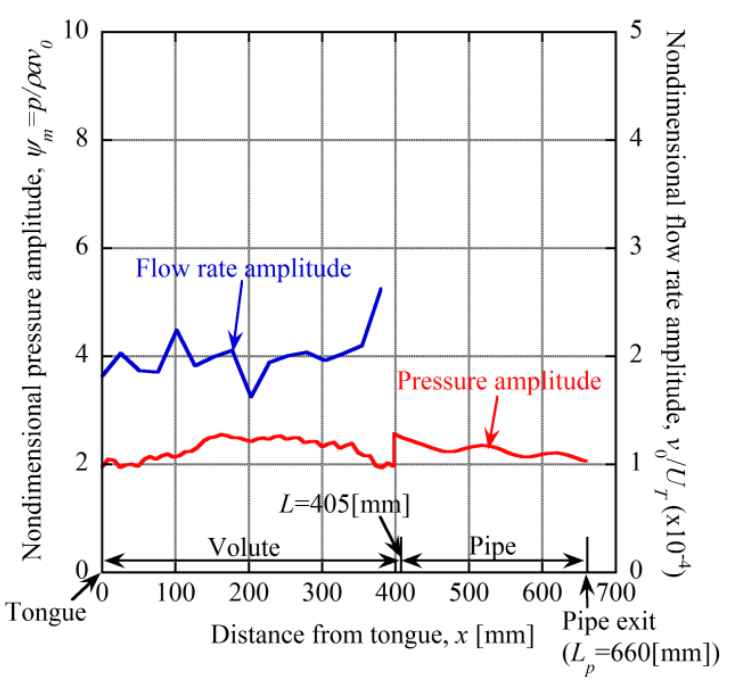

(a) $S=16$, Pump mode

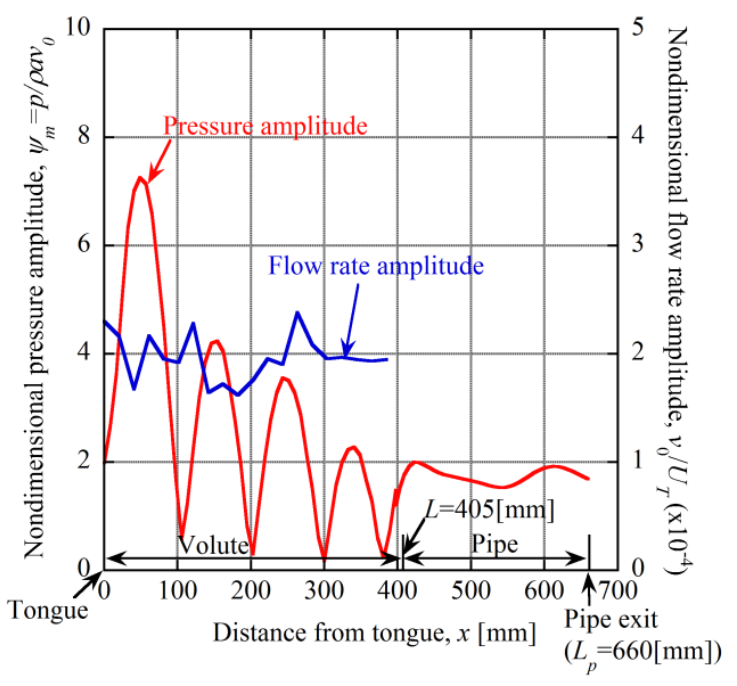

(c) $S=20$, Pump mode

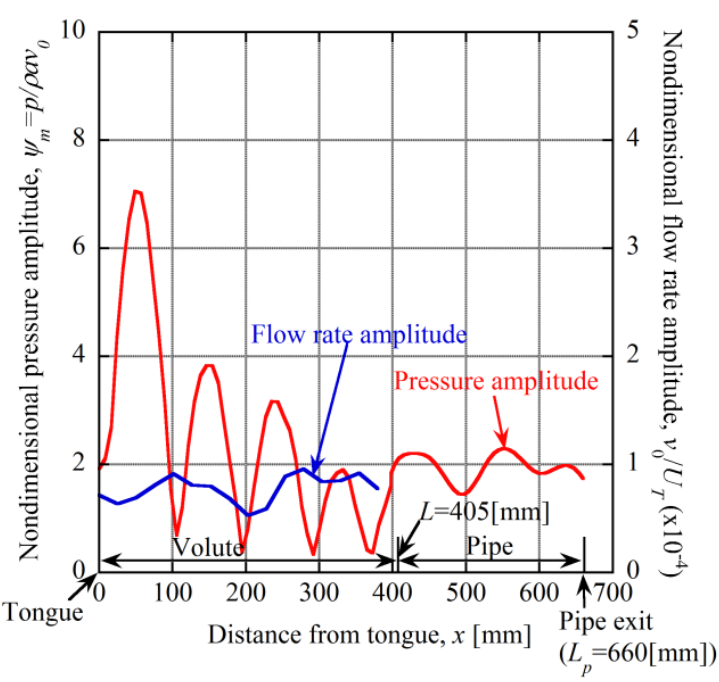

(b) $\mathrm{S}=16$, Turbine mode

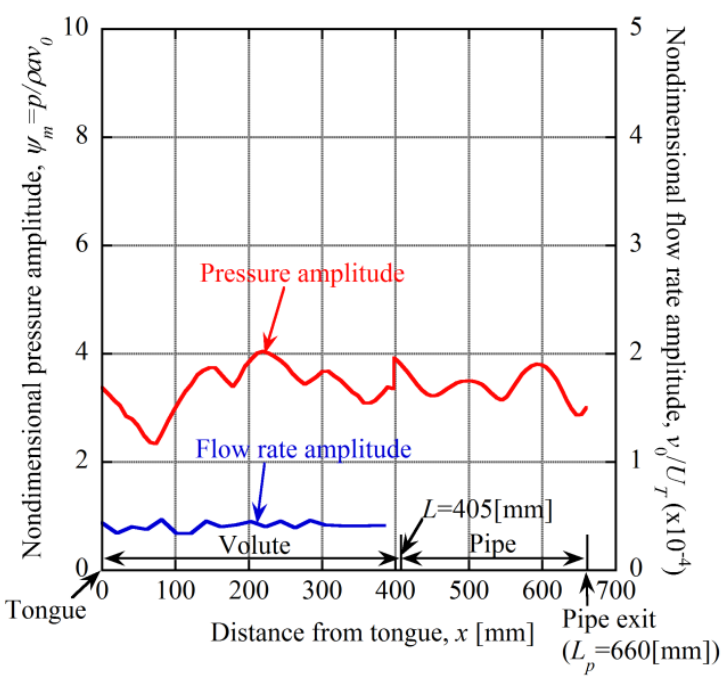

(d) $S=20$, Turbine mode

Fig. 15 Amplitude of pressure and flow rate fluctuation

\section{Non-Dimensional Pressure Using the Radial Velocity at the Stator Outer Radius}

Figure 15 shows the non-dimensional pressure $p / \rho a v_{0}$ using the radial velocity fluctuation $v_{0}$ obtained from CFD. Depending on the direction of the interaction mode, similar results are obtained for pump and turbine modes. This shows that the radial velocity fluctuation $v_{0}$ caused by rotor-stator interaction plays an important role in phase resonance, as assumed in the 1-D model.

\section{Conclusion}

The results of the present study can be summarized as follows.

(1) In phase resonance, the rotational direction of the rotor-stator interaction mode is more important than the rotational direction of the rotor.

(2) Phase resonance can be simulated by careful application of commercial CFD software for compressible unsteady flows.

(3) The pressure fluctuation in the connecting pipe was larger for the pump mode for the case with the stator number $S=16$ but they were of the same level for $S=20$. So, we cannot say for which mode the pressure fluctuation is larger.

(4) The blade passing component of the pressure fluctuation is larger at the inner radius of the stator. The rotor-stator interaction component is of the same order at the inner and outer radii of the stator.

(5) The blade passing frequency component of the flow rate fluctuation through stator blade channel can be evaluated from the pressure fluctuation at the inner radius of the stator.

(6) The amplitude of radial velocity fluctuation caused by rotor-stator interaction plays an important role in phase resonance. The non-dimensional pressure fluctuation normalized by using the radial velocity was nearly the same for pumping and turbine modes. 
We tried to evaluate the pressure fluctuation in the connecting pipe from 1-D model using the radial velocity fluctuation obtained from incompressible CFD. This resulted in the pressure fluctuation about 3 times the experimental results. This method can be used for the quick evaluation of the pressure amplitude under phase resonance.

\section{Nomenclature}

\begin{tabular}{|c|c|c|c|}
\hline$a$ & Velocity of sound $[\mathrm{m} / \mathrm{s}]$ & $S$ & Stator blade number \\
\hline$D_{2}, D_{T}$ & Outer diameter of impeller $[\mathrm{mm}]$ & $U$ & Propagation velocity $[\mathrm{m} / \mathrm{s}]$ \\
\hline$D_{4}$ & Outer diameter of stator $[\mathrm{mm}]$ & $U_{T}$ & Tip speed of impeller $[\mathrm{m} / \mathrm{s}]$ \\
\hline$f$ & Frequency $[\mathrm{Hz}]$ & $v_{0}$ & Radial velocity fluctuation due to RSI [m/s] \\
\hline$j$ & Complex unit & $\Omega$ & Rotating angular frequency of impeller $[\mathrm{rad} / \mathrm{s}]$ \\
\hline$m$ & Circumferential mode number & $\omega$ & Angular frequency $[\mathrm{rad} / \mathrm{s}]$ \\
\hline$L_{p}$ & Distance between volute tongue and pipe exit [m] & $x$ & Distance from volute tang $[\mathrm{m}]$ \\
\hline$M$ & Mach number & $\tilde{\phi}$ & Normalized flow rate fluctuation amplitude through \\
\hline$n$ & Order of harmonics & & stator \\
\hline$p$ & Pressure disturbance $[\mathrm{Pa}]$ & $\Phi$ & Flow coefficient of fan $\left(\Phi=Q /\left(U_{T} D_{T}^{2}\right)\right)$ \\
\hline$\tilde{p}$ & Pressure fluctuation amplitude $[\mathrm{Pa}]$ & $\psi$ & Pressure coefficient \\
\hline$Q$ & Flow rate $\left[\mathrm{m}^{3} / \mathrm{s}\right]$ & $\psi_{e}$ & Non-dimensional pressure amplitude \\
\hline$R$ & Impeller blade number & $\psi_{m}$ & Non-dimensional pressure amplitude from 1-D model \\
\hline
\end{tabular}

\section{References}

[1] Den Hartog J.P., 1929, "Mechanical Vibrations in Penstocks of Hydraulic Turbine Installations," Transactions of ASME.

[2] Y.N. Chen, 1961, "Wasserdruckschwingungen in Spiralgeh ä usen von Speicherpumpen," Suzer-Forschungsheft.

[3] P. Doerfler, 1984, "On the role of phase resonance in vibrations caused by blade passage in radial hydraulic turbomachines," proceedings of IAHR SYMPOSIUM.

[4] Y. Ohura, M. Fujii, O. Sugimoto, H. Tanaka, I. Yamamoto, 1990, "Vibration of the powerhouse structure of a pumped storage power plant," proceedings of IAHR SYMPOSIUM.

[5] Nishiyama, Y., Suzuki, T., Yonezawa, K., Tanaka, H., Doerfler, P., and Tsujimoto, Y., "Phase Resonance in a Centrifugal Compressor," International Journal of Fluid Machinery and Systems, Vol.4, No.3,July-September, 2011, pp. 325-333

[6] Tsujimoto, Y., Tanaka, H., Doerfler, P., Yonezawa, K., Suzuki, T., and Makikawa, K., Effects of Acoustic Resonance and Volute Geometry on Phase Resonance in a Centrifugal Fan, to be published in IJFMS.

[7] Makey, E., Cooper, P., Sloteman, D.P., Gibson, R., 1993, "Investigation of Pressure Pulsations Arising from Impeller/Diffuser Interaction in a Large Centrifugal Pump," Rotating Machinery: Problems \& Solutions.

[8] Parmakian, J., Vibration of the Grand Coulee Pump-Discharge Lines, Trans. ASME, July 1954.

[9] T.J. Poinsot, and S.K. Lele, 1992, "Boundary Condition for Direct Simulations of Compressible Viscous Flows,” Journal of Computational Physics 101, 104-129. 